\title{
Increased CDC20 expression is associated with development and progression of hepatocellular carcinoma
}

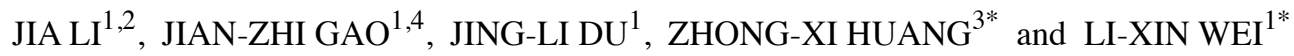 \\ ${ }^{1}$ Department of Pathology, Chinese PLA General Hospital, Beijing 100853; ${ }^{2}$ Department of Clinical Medicine, \\ Medical College, Nankai University, Tianjing 300071; ${ }^{3}$ Cancer Institute, Southern Medical University, \\ Guangzhou, Guangdong 510515; ${ }^{4}$ Department of Basic Medical Sciences, \\ Xinxiang Medical College, Xinxiang 453000, P.R. China
}

Received February 13, 2014; Accepted April 22, 2014

DOI: $10.3892 /$ ijo.2014.2559

\begin{abstract}
Cell division cycle 20 (CDC20) encodes a regulatory protein interacting with the anaphase-promoting complex/cyclosome $(\mathrm{APC} / \mathrm{C})$ in the cell cycle and plays important roles in tumorigenesis and progression of multiple tumors. The present study aimed to investigate the clinical significance of CDC20 in hepatocellular carcinoma (HCC) and the role of CDC20 in the progression of HCC. By bioinformatics analysis, CDC20 was found to be the major node in HCC molecular interaction networks. Quantitative PCR and western blot analyses were applied to examine CDC20 expression in 16 paired primary HCC tissues. Immunohistochemistry (IHC) was performed to examine CDC20 protein expression in 132 matched paraffinembedded HCC tissues and to analyze the relationship between CDC20 staining and clinical characteristics. Small interfering RNA (siRNA) targeting CDC20 was synthesized and transfected into HepG2 cells to investigate the role of CDC20 in cell growth and the cell cycle. Results show that CDC20 expression was upregulated in HCC tissues compared to adjacent non-tumor liver tissues. In the 132 matched HCC tissues, high expression levels of CDC20 were detected in $68.18 \%$ HCC samples, and overexpression of CDC20 was positively correlated with gender $(\mathrm{P}=0.013)$, tumor differentiation $(\mathrm{P}=0.000)$, TNM stage $(\mathrm{P}=0.012), \mathrm{P} 53$ and Ki-67 expression $(\mathrm{P}=0.023$ and $\mathrm{P}=0.007$, respectively). Cells transfected with $\mathrm{CDC} 20$ siRNA showed a decrease in cell proliferation and increase in the number of cells in G2/M-phase. In conclusion, increased expression of CDC20 was demonstrated to be associated with the development and progression of $\mathrm{HCC}$, and may be regarded as a promising therapeutic target for HCC.
\end{abstract}

Correspondence to: Professor Li-Xin Wei, Department of Pathology, Chinese PLA General Hospital, Beijing 100853, P.R. China E-mail: weilx301@263.net

*Contributed equally

Key words: cell division cycle 20, hepatocellular carcinoma, clinical characteristics, cell proliferation, cell cycle

\section{Introduction}

Hepatocellular carcinoma (HCC) which represents the major histological subtype of primary liver cancers, is the fifth most frequent cancer worldwide and the third leading cause of cancer mortality $(1,2)$. The highest liver cancer rates are found in developing countries especially in East/South-East Asia and Middle/ Western Africa, whereas rates are low in South-Central, Western Asia, Northern and Eastern Europe (3). Genetic alteration and epigenetic high-risk factors such as chronic infection with HBV or HCV, hepatic cirrhosis, alcoholic liver disease and aflatoxins account for the high morbidity of HCC (4-6). However, the molecular mechanism accompanied by hepatocarcinogenesis and progression is still largely unclear. Thus, it is critical for us to clarify the etiology and investigate the vitally molecular alteration underlying hepatocellular carcinoma initiation and progression, and ultimately improve our current concepts for diagnosing, screening and treatment of this disease.

During the process of hepatocarcinogenesis, the abrogation of cell-cycle checkpoints is an important hallmark that may promote cancer formation (2). As one of the regulators of the cell cycle checkpoint, cell division cycle 20 (CDC20) appears to act as a regulatory protein interacting with the anaphasepromoting complex/cyclosome (APC/C) in the cell cycle, which is required for anaphase initiation and late mitosis exit $(7,8)$. Two regulatory factors, $\mathrm{CDC} 20$ and $\mathrm{CDH} 1$ directly bind to $\mathrm{APC}$ and activate its cyclin ubiquitination activity during mitosis and G1 phase $(9,10)$. It has been reported that the receptor-associated protein 80 (RAP80), which recruits BRCA1 to DNA damage sites in the ubiquitin signaling pathway induced by DNA damage, can be degraded by the anaphase-promoting complex (APC/C-Cdc20) or (APC/C-Cdh1) through polyubiquitination during mitosis to the G1 phase (11). The depletion of RAP80 showed a defective control of G2-M phase checkpoint and promoted mitotic cell cycle progression.

CDC20 expression can be remarkably suppressed by p53 protein which inhibits malignant transformation through regulation of the cell cycle, cellular senescence and apoptosis related genes (12). High expression of CDC20 has been reported in various malignant tumors including pancreatic ductal adenocarcinoma (13), oral squamous cell carcinoma, gastric cancer (14), cervical cancer (15) and various cancer 
cells $(14,16)$. However, the expression pattern of CDC20 and its clinical significance in human hepatocellular carcinoma have not been clarified.

In this study, by analyzing the microarray dataset (accession no. GSE14520) from Gene Expression Omnibus (GEO) database (http://www.ncbi.nlm.nih.gov/geo/), we found that CDC20 was the major node in HCC molecular interaction networks. We examined the expression level of CDC20 in primary HCC and adjacent non-tumor tissues, and evaluated its clinicopathologic significance in 132 archived HCC samples. The effect of the knockdown of CDC20 by siRNA on the growth and cell cycle of liver cancer cells was also investigated. Our findings suggest that CDC20 may play a significant role in the development of HCC.

\section{Materials and methods}

Bioinformatics analysis. Microarray dataset GSE14520 (17) was downloaded from GEO. A total of 183 HCC and 179 corresponding para-carcinoma tissues that were assayed with Affymetrix U133A GeneChips from cohort 2 Chinese patients were used in this study. Gene expression profiling data was re-summarized using the RMA method (18) and Entrez gene-centric CDF files (19) (instead of original Affymetrix CDF files), which filtered non-specific probes on the GeneChips and merged multiple probe sets representing the same Entrez gene into one probe set. Significance analysis of microarray (SAM) (20) was performed to identify differently expressed genes between HCC and corresponding para-carcinoma tissues. Delta was set to 2.25, and the threshold of FDR was set to 0.001. Genes overexpressed in HCC that were expressed in more than $50 \mathrm{HCC}$ tissues but less than 10 corresponding para-carcinoma tissues were studied. The genes were further analyzed with GenCLiP software (21) (http://ci.smu.edu.cn) to annotate gene functions and construct molecular interaction networks.

Ethics statement. The clinical samples were used for research purposes only. Approval was obtained from the ethics committee of Chinese PLA General Hospital (LREC 2012/40). All samples were collected under the condition of a prior written informed consent from the patients.

Patients and tissue samples. Sixteen pairs of fresh HCC and adjacent non-tumor tissues used for real-time PCR and western blot analyses were collected during surgery from the Chinese PLA General Hospital (Beijing, China) from November, 2012 to February, 2013. Tissues were snap-frozen in liquid nitrogen until use. Paraffin-embedded, archived HCC and adjacent non-tumor tissues used for immunohistochemistry (IHC) were obtained from $132 \mathrm{HCC}$ patients who underwent partial liver resection at the same hospital between January, 2006 and December, 2007. The median age of the patients was 52 years (range 24-80 years).

Cell culture. One normal liver cell line (LO2) and three HCC cell lines (HepG2, SMMC7721, Huh7) were purchased from the American Type Culture Collection (ATCC, Manassas, VA) or Chinese Academy of Science Cell Bank and were maintained in the Institute of Biotechnology, Academy of Military Medical Sciences. All cells were maintained in Dulbecco's modified Eagle's medium (DMEM, Invitrogen, CA) supplemented with $10 \%$ fetal bovine serum (FBS, Gibco, Carlsbad, CA) and $2 \mathrm{mM}$ L-glutamine, $100 \mathrm{U} / \mathrm{ml}$ penicillin, $100 \mu \mathrm{g} / \mathrm{ml}$ streptomycin at $37^{\circ} \mathrm{C}$ with $5 \% \mathrm{CO}_{2}$.

RNA extraction, cDNA synthesis and real-time PCR analysis. Total RNA was extracted from cell lines and tissue samples using TRIzol reagent (Invitrogen) according to the manufacturer's instructions. A total of $2 \mu \mathrm{g}$ RNA was reverse transcribed using the TransScript and cDNA Synthesis Kit from TransGen Biotech (Beijing, China). Real-time PCR was performed to examine CDC20 mRNA level in cell lines and tissue samples by using a Bio-Rad iQ5 Multicolor Real-Time PCR Detection System (Bio-Rad, Hercules, CA). $\beta$-actin was used as an internal control for normalization. PCR primers were designed using the Primer Premier 5 software and the sequences were: CDC20 forward, 5'-TCGCATCTGGAATGTGTGCT-3'; and reverse, 5'-CCCGGGATGTGTGACCTTTG-3'; $\beta$-actin forward, 5'-TGACGTGGACATCCGCAAAG-3'; and reverse, 5'-CTGG AAGGTGGACAGCGAGG-3'. Expression data were normalized to the geometric mean of the housekeeping gene $\beta$-actin and calculated with the $\Delta \Delta \mathrm{Ct}$ (22) and results were expressed with $2^{-\Delta \Delta \mathrm{Ct}}$.

Western blot analysis. Western blot analysis was performed under the standard protocol. SDS-polyacrylamide gel electrophoresis $(10 \%)$ was used to separate the protein which was then electrotransferred from the gel to a polyvinylidene fluoride (PVDF) membrane. After blocking with 5\% dried skim milk for $1 \mathrm{~h}$, the membrane was incubated with rabbit anti-human CDC20 polyclonal antibody (1:1,000, Bioworld Technology, St. Louis Park, MN) for $1 \mathrm{~h}$ at room temperature. The mouse anti-human $\alpha$-tubulin monoclonal antibody (1:5,000; Santa Cruz Biotechnology, Santa Cruz, CA) was used as an internal control. After washing with Tris-buffered saline with Tween-20 (TBST) three times, the membrane was incubated with secondary horseradish peroxidase-conjugated antibody against rabbit or mouse (dilution 1:5,000). Chemiluminescent detection was performed with the Immobilon Western Chemiluminescent HRP Substrate kit (Millipore Corporation, Billerica, MA).

Immunohistochemistry (IHC) and scoring. Immunohistochemistry for CDC20 expression in HCC and adjacent non-tumor samples was performed using standard methods. Briefly, tissue sections were incubated with rabbit anti-CDC20 diluted 1:200 (Bioworld Technology) overnight at $4^{\circ} \mathrm{C}$. Bovine serum albumin (1\%; BSA) without primary antibody was used as the negative control. The secondary poly-horseradish peroxidase (HRP) anti-rabbit IgG antibody (ZSGB-Bio, Beijing, China) was incubated in room temperature for $20 \mathrm{~min}$.

All of the immunostained sections were reviewed and scored independently by two pathologists in a blinded manner without knowledge of the clinicopathological information, based on the H-score method, which considers the staining intensity together with the percentage of cells staining positively $(23,24)$. For H-score method, 10 fields were chosen randomly at $x 400$ magnification. The staining intensity in the cells was scored as $0,1,2$ and 3 corresponding to the negative, weak, intermediate and strong staining, respectively. 
In each field the total number of cells and cells stained at each intensity were counted. The H-score was calculated following the formula: ( $\%$ of cells stained at intensity category $1 \times 1)+(\%$ of cells stained at intensity category $2 \times 2)+(\%$ of cells stained at intensity category $3 \times 3)$. H-scores varied from 0 to 300 where 300 represented $100 \%$ of cells strongly stained (3+) (24). High CDC20 expression was defined as staining H-scores of cells $\geq 200$.

Suppression of CDC20 by small interfering RNA (siRNA). Double-stranded, small interfering RNA (siRNA) was synthesized and purified by GenePharma (Shanghai, China). The subsequences corresponding to the coding region of human CDC20 were: sense, 5'-GGAGCUCAUCUCAGGCCA UUU-3'; antisense, 5'-AUGGCCUGAGAUGAGCUCCUU-3'. A scrambled non-targeting siRNA was used for the negative control. These siRNAs were dissolved in diethyl pyrocarbonate (DEPC) water to reach a concentration of $20 \mu \mathrm{M}$. Liver cancer HepG 2 cells were treated with CDC20 siRNA or negative control siRNA in $20 \mathrm{nM}$ by using the INTERFERin in vitro siRNA transfection reagent (Polyplus Transfection, New York, NY).

Quantitative real-time PCR and western blot analyses were used for detecting the interference effect of siCDC20. Cells that were untreated or treated with negative control siRNA oligonucleotides were the control groups.

Cellular proliferation assay. Two $25-\mathrm{cm}^{2}$ plastic flasks were each inoculated with $2 \times 10^{5}$ liver cancer cells (HepG2), which were then transfected with $20 \mathrm{nM}$ negative control siRNA and CDC20 siRNA, respectively, for 48-h incubation. Then, cells were digested and seeded into 6 -well plates containing $2 \mathrm{ml}$ medium per well. Afterwards, the cells from a well were digested and counted by the automated cell counter (Invitrogen) every $24 \mathrm{~h}$ until day five.

Fluorescence-activated cell sorting (FACS) test of the cell cycle. siRNA for CDC20 and negative control siRNA oligonucleotides were transfected into HepG2 cells with the INTERFERin in vitro siRNA transfection reagent for $48 \mathrm{~h}$. After treatment with $2 \mu \mathrm{g} / \mathrm{ml}$ thymidine (Sigma-Aldrich, St. Louis, MO) for $24 \mathrm{~h}$, cells were harvested at $0,3,6,9$, 12-h after removing thymidine from the medium and fixed with $70 \%$ alcohol. Prior to analyses, cells were washed with PBS, treated with $1 \mathrm{mg} / \mathrm{ml}$ RNase A at $37^{\circ} \mathrm{C}$ for $30 \mathrm{~min}$ and then stained with propidium iodide (PI). Analyses were performed by BD FACSCalibur (Becton-Dickinson, Franklin Lakes, NJ) and results were analyzed by WinMDI Version 2.9 software.

Statistical analyses. All statistical analyses were made using the IBM SPSS 20.0 statistical software package. One-way analysis of variance (ANOVA) was used to compare the expression of CDC20 mRNA normalized to $\beta$-actin in cell lines. The same method was also performed in comparing the histological differentiation in tumor tissues normalized to normal liver samples. Data comparisons in two groups were performed by Student's t-test. The $\chi^{2}$ test was used to analyze the relationship between CDC20 expression and clinicopathological features. Bivariate correlations between variables were calculated by Spearman's correlation coefficients. The level of statistic significance was set at $\mathrm{P}<0.05$ for all tests.

\section{Results}

CDC20 is the major node in HCC molecular interaction networks. SAM analysis identified 4,358 genes overexpressed in $\mathrm{HCC}$, in which 137 genes were expressed in more than 50 HCC tissues, but in less than 10 para-carcinoma tissues. GenCLiP analysis showed that among the 137 genes, 72 genes were related to the cell cycle $\left(\mathrm{P}=1.238 \mathrm{e}-15\right.$, by $\chi^{2}$ test $)$, 19 genes were related to spindle assembly $(\mathrm{P}=2.172 \mathrm{e}-55$, by $\chi^{2}$ test), and 26 genes were related to chromosome segregation $\left(\mathrm{P}=5.472 \mathrm{e}-55\right.$, by $\chi^{2}$ test). Among the molecular networks constructed with the 137 genes, CDC20 was the major node that has not been previously reported to be related with HCC. Nine genes (NEK2, E2F1, BUB1, BUB1B, AURKB, CCNB1, CCNB2, UBE2C and CDKN2A) are known to interact with CDC20, in which 7 genes were related with spindle assembly checkpoint (SAC) (Fig. 1). The target of the SAC is CDC20 (25). Thus, we inferred that in HCC, overexpression of CDC20 leads to absence of SAC, and cells rapidly become aneuploid.

CDC20 is overexpressed in HCC. Quantitative real-time PCR was used to examine transcript level of CDC20 in three HCC cell lines (HepG2, SMMC-7721 and Huh7), and one normal liver cell line (LO2). CDC20 mRNA level was higher in all the three HCC cell lines than that in the normal cell line (Fig. 2).

In order to determine whether the upregulation of CDC20 in HCC cell lines is similar in HCC patients, we performed quantitative real-time PCR on 16 pairs of matched HCC and adjacent normal liver tissues. As shown in Fig. 3A, CDC20 was found to be differentially overexpressed in 14 of all examined human primary HCC samples compared with non-cancerous samples from the same patients. Additionally, the tumor/non-tumor (T/NT) ratio of CDC20 mRNA expression was at least $>2$-fold in all these 14 upregulated samples, and the highest ratio was even up to about 40-fold. The protein level of CDC20 was confirmed in all the 16 paired tissue samples by western blot analysis. Image $\mathrm{J} 1.47 \mathrm{v}$ software was used to quantize the grey level of each band and calculate the CDC20 T/NT ratio of each patient normalizing with $\alpha$-tubulin. Results revealed that all examined HCC samples showed a higher expression level of CDC20 protein except sample 1 and sample 4, which was consistent with the mRNA level (Fig. 3B). These findings indicated that $\mathrm{CDC} 20$ was commonly upregulated in either HCC tissues or cell lines.

Immunohistochemistry staining of CDC20 protein. Immunohistochemistry (IHC) was performed to analyze the protein expression and localization of CDC20 in 132 paraffin-embedded archived HCC tissue samples, including 14 cases of well differentiated, 78 cases of moderate differentiated and 40 cases of poor differentiated tumors. CDC20 protein was upregulated (H-score $\geq 200)$ in $90(68.18 \%)$ of 132 HCC tissues. As shown in Fig. 4A, high levels of CDC20 were present in primary HCC lesions and the positive staining mainly localized in 


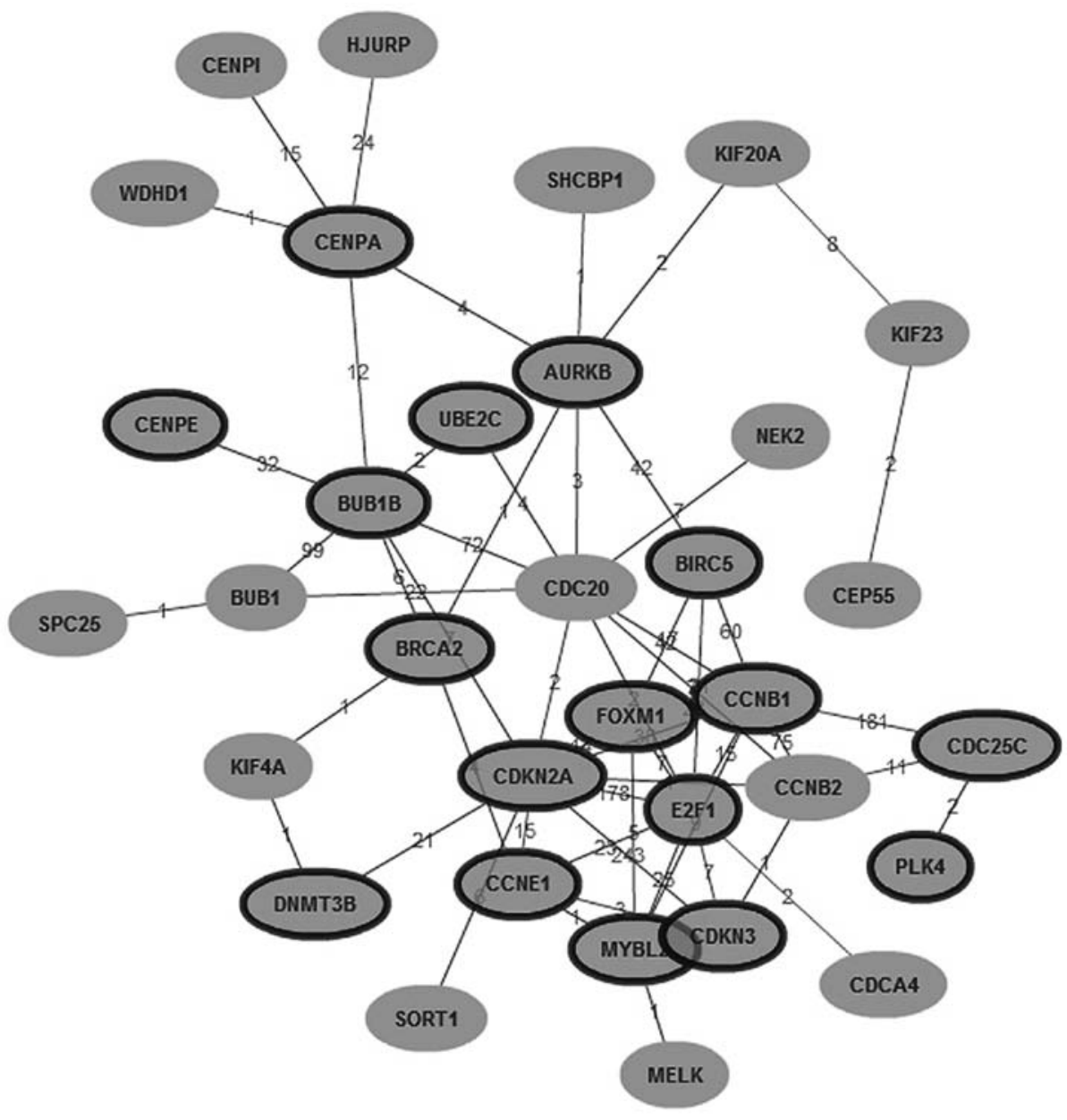

Figure 1. Molecular interaction network constructed with the 137 HCC overexpressed genes. The node with black border represents known HCC-related genes; the number on each edge indicates number of papers reporting the interaction of the two genes.

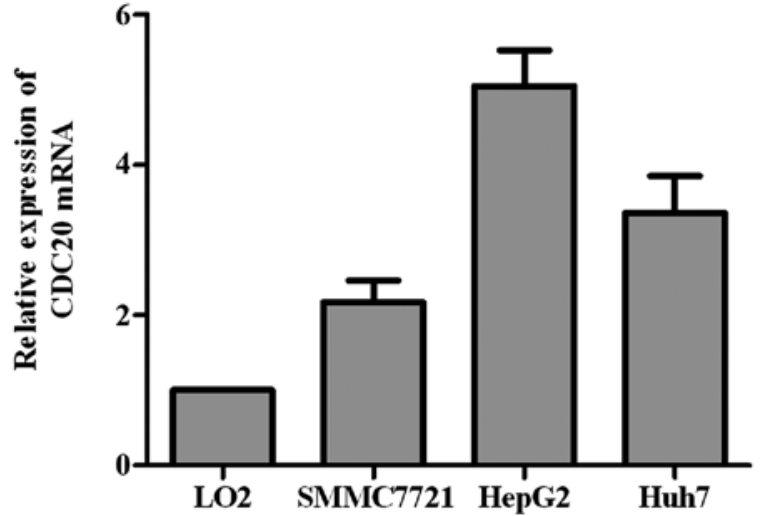

Figure 2. Expression analysis of CDC20 in cell lines. Analyzing CDC20 mRNA expression in normal liver cell line LO2 and HCC cell lines (HepG2, SMMC7721, Huh7) by real-time reverse transcription PCR. Expression data were normalized by $\beta$-actin and analyzed with one-way analysis of variance (ANOVA). ${ }^{*} \mathrm{P}=0.002$.

cytoplasm and nuclei. In contrast, CDC20 was negatively or weakly stained in corresponding non-tumor tissues. By quantitatively comparing the scores of CDC20 staining between 132 archived HCC and adjacent non-tumor tissues (mainly including hepatocirrhosis and hepatitis), the scores of CDC20 staining was significantly increased in HCC samples compared to peritumoral samples (Fig. 4B). In addition, the scores of CDC20 staining was significantly increased along with the progression of tumor histological differentiation from well to poorly differentiated tissues (Fig. 5).

Correlation between increased CDC2O expression and clinicopathological parameters of HCC. The relationship between CDC20 protein expression and the clinicopatholigical features of $132 \mathrm{HCC}$ patients was further analyzed. As summarized in Table I, CDC20 expression was significantly associated with gender $(\mathrm{P}=0.013)$, tumor differentiation $(\mathrm{P}=0.000)$, TNM stage $(\mathrm{P}=0.012), \mathrm{P} 53$ and $\mathrm{Ki}-67$ expression $(\mathrm{P}=0.023$ and $\mathrm{P}=0.007$, respectively). However, no statistically significant association was found between high CDC20 expression and other clinicopathological characteristics including age, tumor size, HBV infection, serum AFP level, hepatic cirrhosis, vascular invasion and intra/extra hepatic metastasis. Spearman correlation analysis revealed that high expression of CDC20 was closely related with gender $(\mathrm{R}=0.215, \mathrm{P}=0.013)$, poorer tumor differentiation $(\mathrm{R}=0.421$, $\mathrm{P}<0.001)$, advanced TNM staging $(\mathrm{R}=0.218, \mathrm{P}=0.012)$, higher expression level of $\mathrm{p} 53(\mathrm{R}=0.212, \mathrm{P}=0.014)$ and $\mathrm{Ki}-67$ $(\mathrm{R}=0.235, \mathrm{P}=0.007)$ (Table II). Taken together, these results 

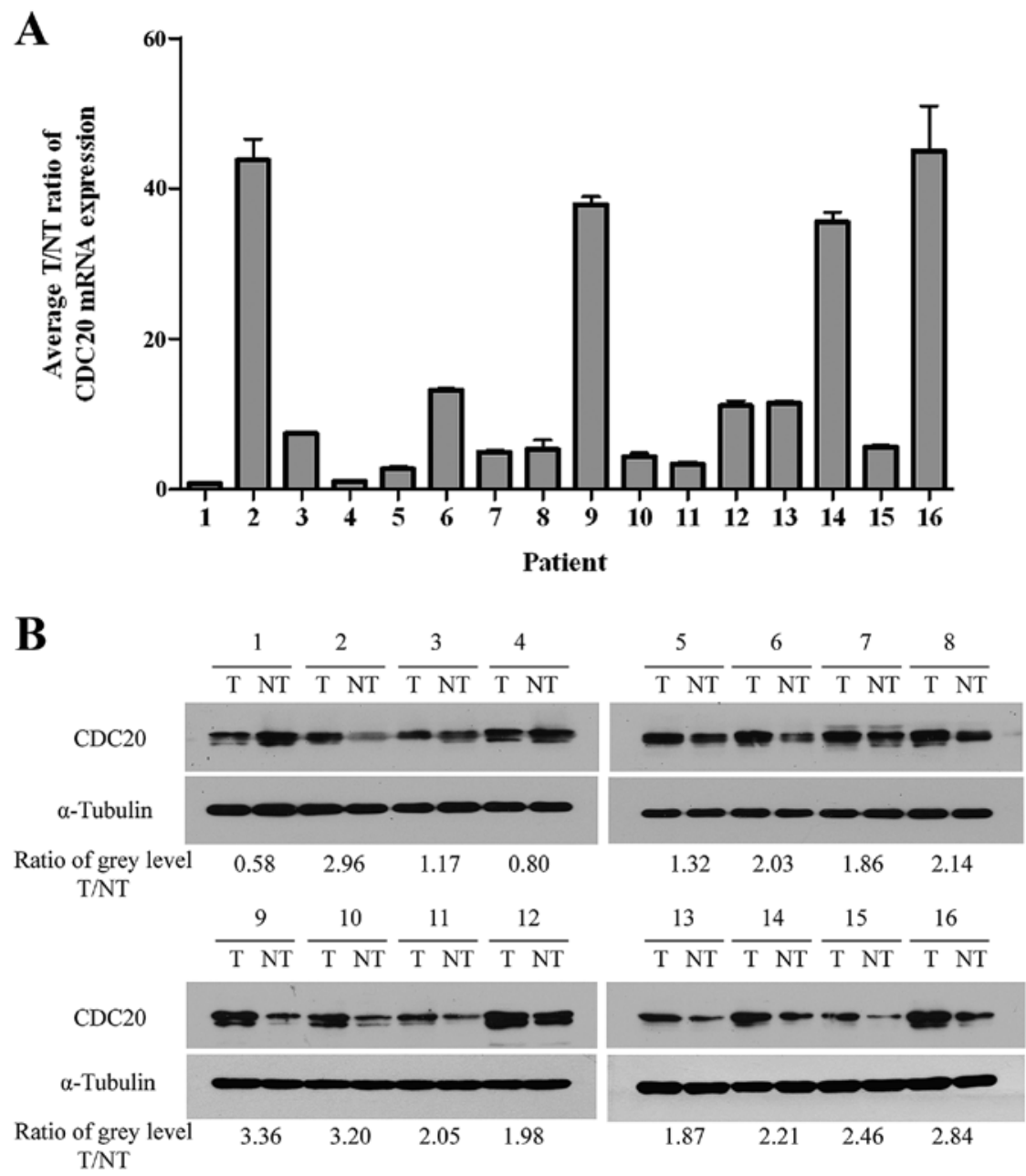

Figure 3. Expression of CDC20 in fresh frozen HCC tissues. Expression of CDC20 mRNA and protein level in 16 pairs of HCC (T) and adjacent non-tumor tissues (NT) detected by (A) real-time PCR and (B) western blot analysis.

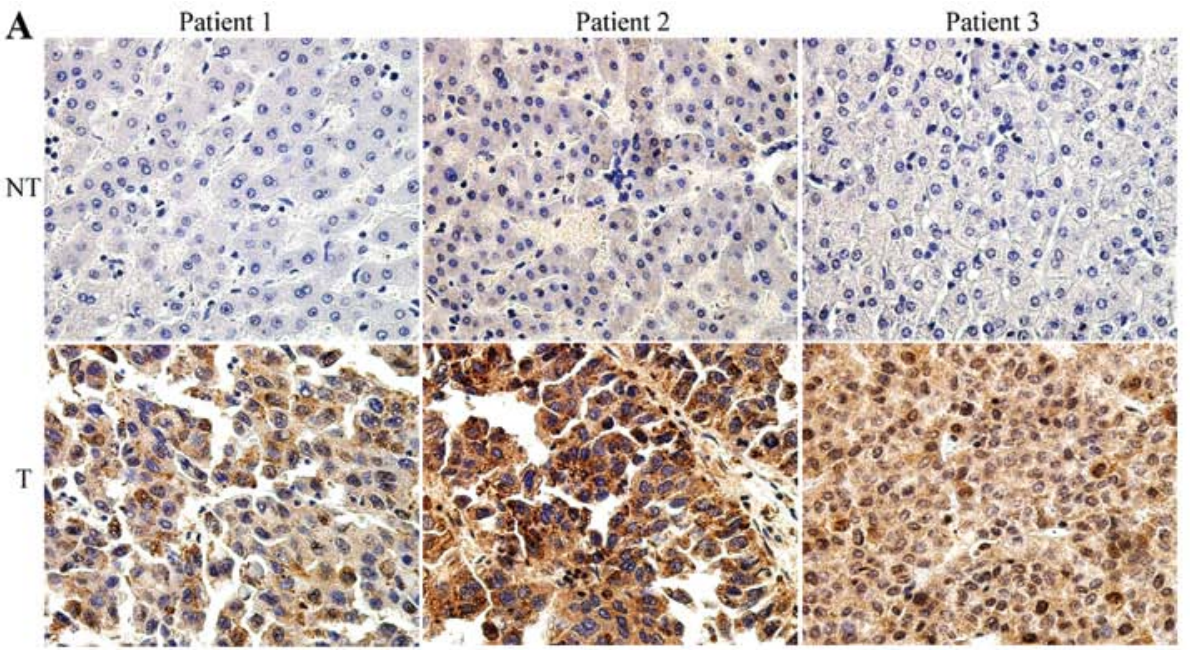

B

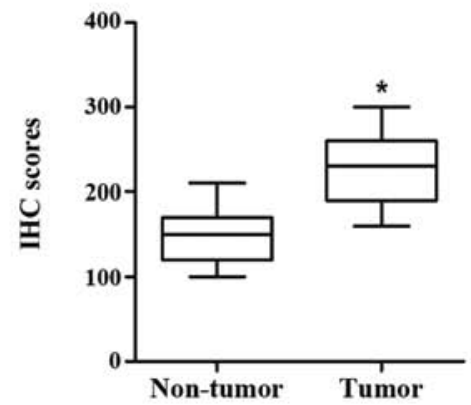

Figure 4. Immunohistochemistry (IHC) staining of CDC20 in HCC tissues. (A) IHC staining of CDC20 protein in paraffin-embedded HCC and adjacent non-tumor tissues (x400). (B) Statistical analysis of the average score of CDC20 staining between HCC tissues and corresponding non-tumor tissues. " $\mathrm{P}<0.05$ (Student's t-test).

indicated that CDC20 was highly expressed in HCC tissues and its expression closely correlated with the differentiation and progression of HCC.
Suppression of CDC2O expression by siRNA. The quantitative real-time PCR revealed $90 \%$ transcriptional level suppression of CDC20 at $48 \mathrm{~h}$ after transfection with 


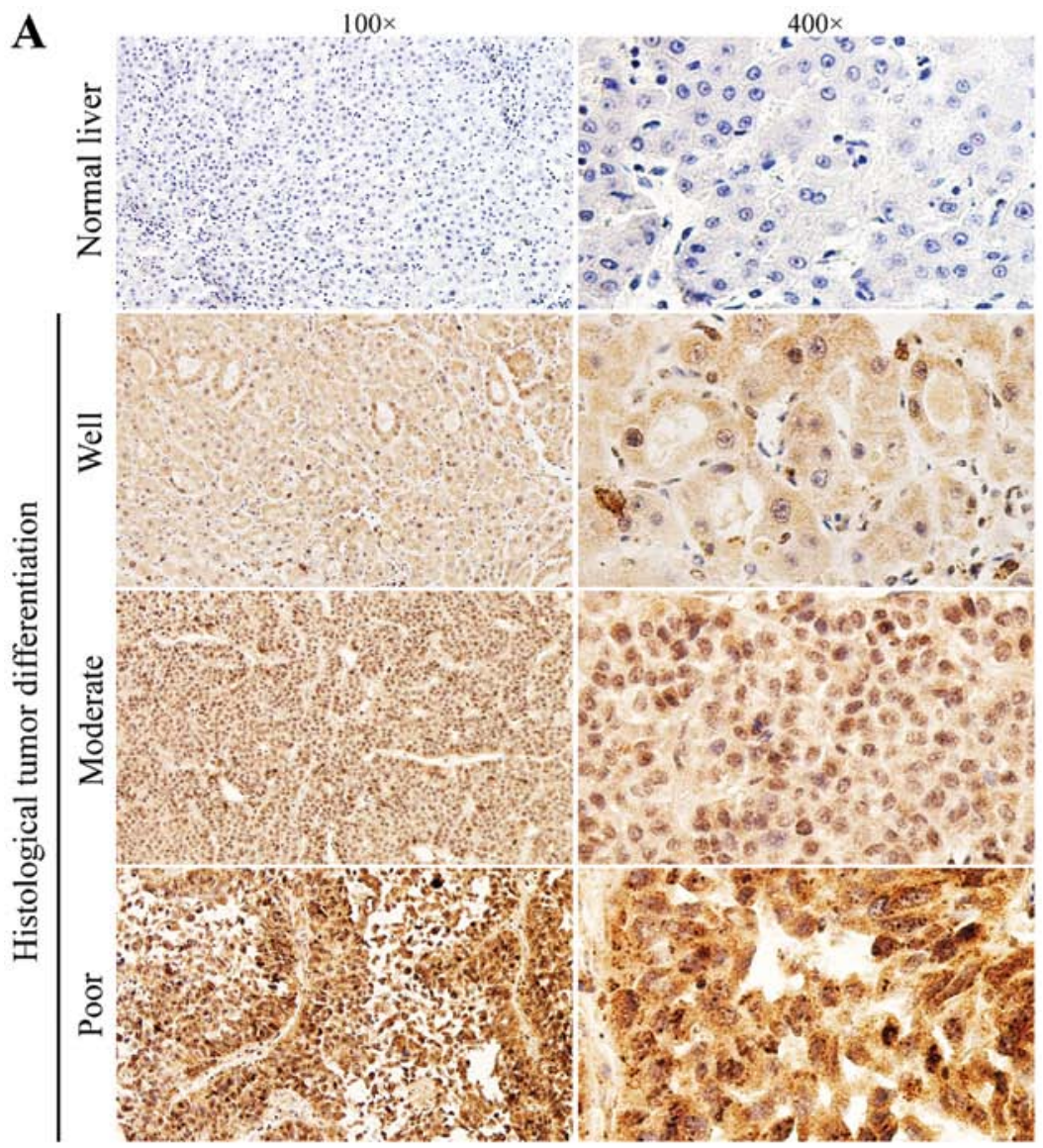

B

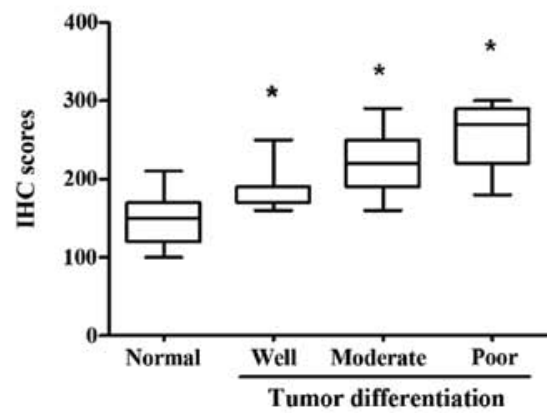

Figure 5. Immunohistochemistry (IHC) staining of CDC20 in poor, moderate and well-differentiated HCC tissues. (A) Representative images from immunohistochemistry analyses of CDC20 expression in normal liver tissue and different histological differentiation of HCC tissues. (B) Statistical analyses of the average score of CDC20 staining between normal liver tissues and HCC tissues of different histological differentiation. " $\mathrm{P}<0.05$ (one-way ANOVA).

siCDC20, comparing with untreated cells or cells transfected with negative control siRNA (Fig. 6A) $(\mathrm{P}<0.0001)$. The western blot analysis also showed an obvious suppression of CDC20 protein level at $48 \mathrm{~h}$ after transfection with siCDC20 (Fig. 6B). The altered expression of cyclin-dependent kinase inhibitor $1 \mathrm{~A}(\mathrm{p} 21)$ protein was also examined by western blot analysis, and the results showed that $\mathrm{P} 21$ protein level was remarkably upregulated owing to the suppression of CDC20 (Fig. 6B).

Effect of CDC20 suppression on cell proliferation and the cell cycle. Cell proliferation assay revealed that the cell growth of CDC20 siRNA transfected cells was remarkably inhibited compared to the cells transfected with negative control siRNA (Fig. 6C). The inhibition of cell proliferation by siCDC20 was expected to be accompanied by the arrest at metaphase of the cell cycle. By flow cytometry test, an increased number of cells in the G2/M phase was observed in siCDC20 transfected cells compared to cells transfected with negative control siRNA (Fig. 6D). These data indicated that suppression of $\mathrm{CDC} 20$ inhibited cell growth by inducing $\mathrm{G} 2 / \mathrm{M}$ phase arrest.

\section{Discussion}

Through bioinformatics analysis of a microarray dataset from GEO database, we identified CDC20 which was the major node in HCC molecular interaction networks, it was related with spindle assembly checkpoint (SAC) in cell cycle. During tumorigenesis, defects or disruption in the spindle assembly 
Table I. Correlations between high CDC20 expression and clinicopathological parameters in HCC patients.

\begin{tabular}{|c|c|c|c|c|c|}
\hline \multirow[b]{2}{*}{ Characteristics } & \multirow[b]{2}{*}{$\mathrm{n}=132$} & \multicolumn{2}{|c|}{ CDC20 expression } & \multirow[b]{2}{*}{$\chi^{2}$} & \multirow[b]{2}{*}{ P-value } \\
\hline & & High $(\geq 200)$ & Low $(<200)$ & & \\
\hline Gender & & & & 6.115 & $0.013^{\mathrm{a}}$ \\
\hline Male & 102 & 64 & 38 & & \\
\hline Female & 30 & 26 & 4 & & \\
\hline Age (years) & & & & 3.683 & 0.162 \\
\hline$<40$ & 22 & 16 & 6 & & \\
\hline $40-60$ & 74 & 54 & 20 & & \\
\hline$>60$ & 36 & 20 & 16 & & \\
\hline Tumor size $(\mathrm{cm})$ & & & & 2.500 & 0.114 \\
\hline$\leq 5$ & 56 & 34 & 22 & & \\
\hline$>5$ & 76 & 56 & 20 & & \\
\hline $\mathrm{HBV}$ & & & & 3.132 & 0.077 \\
\hline Positive & 116 & 76 & 40 & & \\
\hline Negative & 16 & 14 & 2 & & \\
\hline Serum AFP $(\mu \mathrm{g} / \mathrm{l})$ & & & & 2.084 & 0.149 \\
\hline$<400$ & 76 & 48 & 28 & & \\
\hline$\geq 400$ & 56 & 42 & 14 & & \\
\hline Peritumoral tissue & & & & 3.067 & 0.080 \\
\hline Cirrhosis & 106 & 76 & 30 & & \\
\hline Non-cirrhosis & 26 & 14 & 12 & & \\
\hline Vascular invasion & & & & 0.076 & 0.783 \\
\hline Positive & 14 & 10 & 4 & & \\
\hline Negative & 118 & 80 & 38 & & \\
\hline Intra/extra hepatic metastasis & & & & 0.629 & 0.428 \\
\hline Positive & 24 & 18 & 6 & & \\
\hline Negative & 108 & 72 & 36 & & \\
\hline Tumor differentiation & & & & 27.605 & $0.000^{\mathrm{a}}$ \\
\hline Well & 14 & 2 & 12 & & \\
\hline Moderate & 78 & 52 & 26 & & \\
\hline Poor & 40 & 36 & 4 & & \\
\hline TNM stage & & & & 6.286 & $0.012^{\mathrm{a}}$ \\
\hline I-II & 110 & 70 & 40 & & \\
\hline III-IV & 22 & 20 & 2 & & \\
\hline P53 & & & & 7.550 & $0.023^{\mathrm{a}}$ \\
\hline- & 58 & 34 & 24 & & \\
\hline$\leq 50 \%+$ & 52 & 36 & 18 & & \\
\hline$>50 \%+$ & 22 & 20 & 2 & & \\
\hline $\mathrm{Ki}-67$ & & & & 7.266 & $0.007^{\mathrm{a}}$ \\
\hline$\leq 50 \%+$ & 100 & 62 & 38 & & \\
\hline$>50 \%+$ & 32 & 28 & 4 & & \\
\hline
\end{tabular}

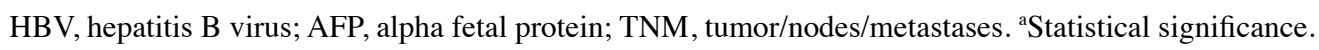

checkpoint were thought to be responsible for the increased rate of aneuploidization (26). Mondal et al have reported that CDC20 is overexpressed in primary head and neck tumors and several oral squamous cell carcinoma (OSCC) cell lines. High expression level of CDC20 in OSCC cells is associated with premature anaphase promotion, leading to mitotic abnor- malities (27). Studies have also revealed that high CDC20 expression is detected in oral squamous cell carcinoma and colorectal cancer tissues and its overexpression may predict a poor prognosis for patients $(28,29)$.

CDC20 is also required for late anaphase cells to exit from mitosis (30). Targeting CDC20 results in blocking of mitosis 


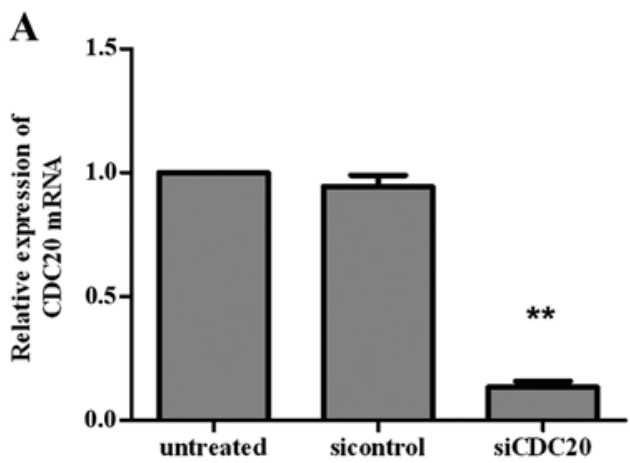

C

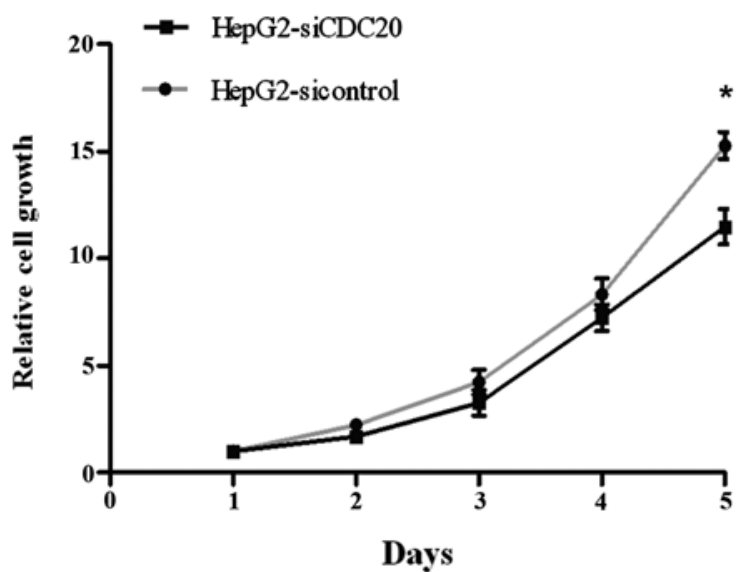

B

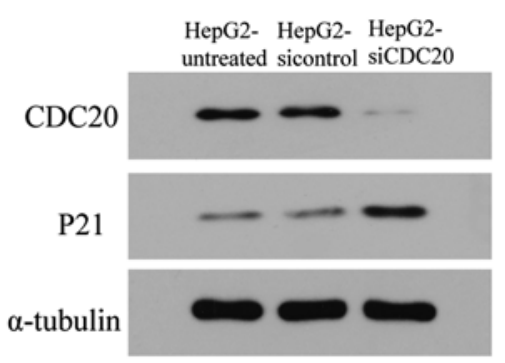

D

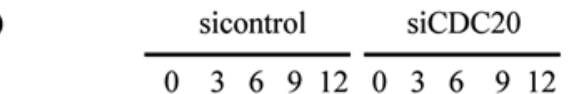

(h)
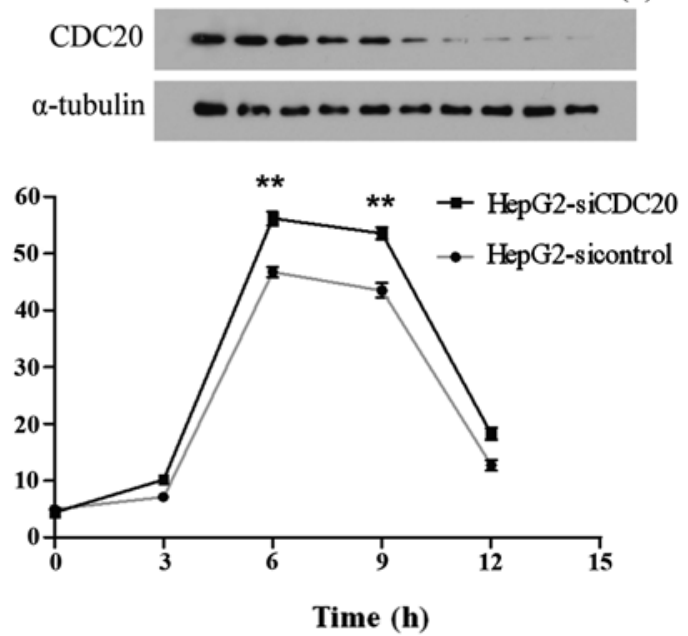

Figure 6. Effect of CDC20 knockdown on the biological characteristics of cells. (A) Examining the suppression of CDC20 mRNA expression level by siRNA in HepG2 cells. Analysis was performed by real-time PCR. * P $<0.01$ (one-way ANOVA). (B) Examining the CDC20 and P21 protein level in HepG2 cells transfected with negative control siRNA and CDC20 siRNA compared with untreated cells by western blotting. (C) Effect of CDC20 knockdown on cell proliferation. HepG2 cells treated with siCDC20 showed a remarkable inhibition in cell growth compared with si-control. "P $<0.05$ (Student's t-test). (D) Effect of CDC20 knockdown on the cell cycle. HepG2 cells transfected with siCDC20 or si-control were treated with $2 \mu \mathrm{g} / \mathrm{ml}$ thymidine for $24 \mathrm{~h}$ to synchronize at S phase. Cells were collected at each time point after release from cell cycle arrest and analysed by western blot and FACS analysis. ${ }^{* *} \mathrm{P}<0.01$ (Student's t-test).

Table II. Spearman correlation analysis between CDC20 expression level and clinicopathological factors.

\section{CDC20 expression}

\begin{tabular}{lcr}
\cline { 2 - 3 } Characteristics & Correlation coefficient & P-value \\
\hline Gender & 0.215 & 0.013 \\
Age (years) & -0.142 & 0.104 \\
Tumor size $(\mathrm{cm})$ & 0.138 & 0.116 \\
HBV & -0.154 & 0.078 \\
Serum AFP $(\mu \mathrm{g} / \mathrm{l})$ & 0.126 & 0.151 \\
Para-cancerous tissue & 0.152 & 0.081 \\
Vascular invasion & 0.024 & 0.785 \\
Intra/extra hepatic & 0.069 & 0.432 \\
metastasis & & \\
Tumor differentiation & 0.421 & $<0.001$ \\
TNM stage & 0.218 & 0.012 \\
P53 & 0.212 & 0.014 \\
Ki-67 & 0.235 & 0.007 \\
\hline
\end{tabular}

exit, which may be a better cancer therapeutic strategy for killing cancer cells more effectively than spindle-perturbing drugs (31). Wang et al reported that CDC20, which may function as an oncoprotein to promote the progression and development of human cancers, represent a promising therapeutic target (32). Though the role of CDC20 in tumorigenesis and prognosis of several human cancers has been deeply researched and confirmed, limited studies have investigated the potential function of CDC20 in the initiation and progression of hepatocellular carcinoma.

In this study, we assessed the expression level of CDC20 in 16 paired fresh frozen $\mathrm{HCC}$ tissues and corresponding non-tumor samples. The results indicated that the mean mRNA and protein level of CDC20 in HCC tissues were much higher than in matched non-tumor tissues. Immunohistochemistry was used for investigating the subcellular location of CDC20 and its relationship with clinical pathological parameters of HCC patients. By using $\chi^{2}$ test, we found that higher CDC20 protein expression level was associated with gender, tumor differentiation, TNM stage, P53 and Ki-67 expression. To investigate the potential biological function and 
molecular mechanism of CDC20 in $\mathrm{HCC}$, we designed a double-stranded, small interfering RNA (siRNA) targeting CDC20 to interfere with its expression level in the HepG2 cell line. By cellular proliferation assay and FACS test, we found that cells transfected with siCDC20 oligonucleotides showed decreased growth speed and increased proportion of cells in $\mathrm{G} 2 / \mathrm{M}$ stage.

The specific knockdown of CDC20 by siRNA showed a suppressed effect against liver cancer cell proliferation in vitro, which indicated that the overexpression of CDC20 might be expected to accelerate cell proliferation and promote tumor initiation and progression of HCC. The liver cancer cells with suppressed CDC20 expression were induced to accumulate in G2/M-phase, which may be responsible for the inhibition of cell growth. Cyclin-dependent kinase inhibitor 1A (p21) which is known to participate in the G2/M checkpoint (33), was proved to be upregulated when the CDC20 expression was specifically suppressed in liver cancer cells. P21 can inhibit the activity of CDKs leading to the accumulation of unphosphorylated $\mathrm{Rb}$ tumor suppressor protein, which inhibits the transcriptional activation of E2F and subsequently prevents entry of the cells into $S$ phase of the cell cycle (34).

In conclusion, this is the first study aimed at examining CDC20 expression in HCC tissues and cell lines, providing a new focus that CDC20 may be a clinically relevant indicator and a promising therapeutic target of HCC. Nevertheless, further studies focused on the molecular mechanisms of CDC20 in the initiation and progression of $\mathrm{HCC}$ and research on the prognostic significance of CDC20 are required.

\section{Acknowledgements}

The authors thank their colleagues from the Institute of Biotechnology of the Academy of Military Medicine Sciences for their technical support.

\section{References}

1. Parkin DM: Global cancer statistics in the year 2000. Lancet Oncol 2: 533-543, 2001.

2. El-Serag HB and Rudolph KL: Hepatocellular carcinoma: epidemiology and molecular carcinogenesis. Gastroenterology 132: 2557-2576, 2007.

3. Jemal A, Bray F, Center MM, Ferlay J, Ward E and Forman D: Global cancer statistics. CA Cancer J Clin 61: 69-90, 2011.

4. Severi T, van Malenstein H, Verslype C and van Pelt JF: Tumor initiation and progression in hepatocellular carcinoma: risk factors, classification, and therapeutic targets. Acta Pharmacol Sin 31: 1409-1420, 2010.

5. Michielsen P and Ho E: Viral hepatitis B and hepatocellular carcinoma. Acta Gastroenterol Belg 74: 4-8, 2011.

6. McGivern DR and Lemon SM: Virus-specific mechanisms of carcinogenesis in hepatitis $\mathrm{C}$ virus associated liver cancer. Oncogene 30: 1969-1983, 2011.

7. Fung TK and Poon RY: A roller coaster ride with the mitotic cyclins. Semin Cell Dev Biol 16: 335-342, 2005.

8. Weinstein J, Jacobsen FW, Hsu-Chen J, Wu T and Baum LG: A novel mammalian protein, p55CDC, present in dividing cells is associated with protein kinase activity and has homology to the Saccharomyces cerevisiae cell division cycle proteins Cdc20 and Cdc4. Mol Cell Biol 14: 3350-3363, 1994.

9. Peters JM: Cell biology: the checkpoint brake relieved. Nature 446: 868-869, 2007.

10. Fang G, Yu H and Kirschner MW: Direct binding of CDC20 protein family members activates the anaphase-promoting complex in mitosis and G1. Mol Cell 2: 163-171, 1998.
11. Cho HJ, Lee EH, Han SH, et al: Degradation of human RAP80 is cell cycle regulated by $\mathrm{Cdc} 20$ and Cdh1 ubiquitin ligases. Mol Cancer Res 10: 615-625, 2012.

12. Kidokoro T, Tanikawa C, Furukawa Y, Katagiri T, Nakamura Y and Matsuda K: CDC20, a potential cancer therapeutic target, is negatively regulated by p53. Oncogene 27: 1562-1571, 2008.

13. Chang DZ, Ma Y, Ji B, et al: Increased CDC20 expression is associated with pancreatic ductal adenocarcinoma differentiation and progression. J Hematol Oncol 5: 15, 2012.

14. Kim JM, Sohn HY, Yoon SY, et al: Identification of gastric cancer-related genes using a cDNA microarray containing novel expressed sequence tags expressed in gastric cancer cells. Clin Cancer Res 11: 473-482, 2005.

15. Espinosa AM, Alfaro A, Roman-Basaure E, et al: Mitosis is a source of potential markers for screening and survival and therapeutic targets in cervical cancer. PloS One 8: e55975, 2013.

16. Ouellet V, Guyot MC, Le Page C, et al: Tissue array analysis of expression microarray candidates identifies markers associated with tumor grade and outcome in serous epithelial ovarian cancer. Int J Cancer 119: 599-607, 2006.

17. Roessler S, Jia HL, Budhu A, et al: A unique metastasis gene signature enables prediction of tumor relapse in early-stage hepatocellular carcinoma patients. Cancer Res 70: 10202-10212, 2010.

18. Irizarry RA, Hobbs B, Collin F, et al: Exploration, normalization, and summaries of high density oligonucleotide array probe level data. Biostatistics 4: 249-264, 2003.

19. Dai M, Wang P, Boyd AD, et al: Evolving gene/transcript definitions significantly alter the interpretation of GeneChip data. Nucleic Acids Res 33: e175, 2005.

20. Tusher VG, Tibshirani R and Chu G: Significance analysis of microarrays applied to the ionizing radiation response. Proc Natl Acad Sci USA 98: 5116-5121, 2001.

21. Huang ZX, Tian HY, Hu ZF, Zhou YB, Zhao J and Yao KT: GenCLiP: a software program for clustering gene lists by literature profiling and constructing gene co-occurrence networks related to custom keywords. BMC Bioinformatics 9: 308, 2008.

22. Adhikary S and Eilers M: Transcriptional regulation and transformation by Myc proteins. Nat Rev Mol Cell Biol 6: 635-645, 2005.

23. Detre S, Saclani Jotti G and Dowsett M: A 'quickscore' method for immunohistochemical semiquantitation: validation for oestrogen receptor in breast carcinomas. J Clin Pathol 48: 876-878, 1995.

24. Früh MPM: EGFR IHC score for selection of cetuximab treatment: Ready for clinical practice? Transl Lung Cancer Res 1: 145-146, 2012.

25. Hwang LH, Lau LF, Smith DL, et al: Budding yeast Cdc20: a target of the spindle checkpoint. Science 279: 1041-1044, 1998.

26. Kops GJ, Weaver BA and Cleveland DW: On the road to cancer: aneuploidy and the mitotic checkpoint. Nat Rev Cancer 5: 773-785, 2005

27. Mondal G, Sengupta S, Panda CK, Gollin SM, Saunders WS and Roychoudhury S: Overexpression of Cdc20 leads to impairment of the spindle assembly checkpoint and aneuploidization in oral cancer. Carcinogenesis 28: 81-92, 2007.

28. Moura IM, Delgado ML, Silva PM, et al: High CDC20 expression is associated with poor prognosis in oral squamous cell carcinoma. J Oral Pathol Med 43: 225-231, 2013.

29. Wu WJ, Hu KS, Wang DS, et al: CDC20 overexpression predicts a poor prognosis for patients with colorectal cancer. J Transl Med 11: 142, 2013.

30. Yeong FM, Lim HH, Padmashree CG and Surana U: Exit from mitosis in budding yeast: biphasic inactivation of the Cdc28-Clb2 mitotic kinase and the role of Cdc20. Mol Cell 5: 501-511, 2000 .

31. Huang HC, Shi J, Orth JD and Mitchison TJ: Evidence that mitotic exit is a better cancer therapeutic target than spindle assembly. Cancer Cell 16: 347-358, 2009.

32. Wang Z, Wan L, Zhong J, et al: Cdc20: a potential novel therapeutic target for cancer treatment. Curr Pharm Des 19: 3210-3214, 2013

33. Bunz F, Dutriaux A, Lengauer C, et al: Requirement for p53 and p21 to sustain G2 arrest after DNA damage. Science 282: 1497-1501, 1998

34. Wells J, Boyd KE, Fry CJ, Bartley SM and Farnham PJ: Target gene specificity of E2F and pocket protein family members in living cells. Mol Cell Biol 20: 5797-5807, 2000. 